University of Wollongong

Research Online

Australian Institute for Innovative Materials -

Papers

Australian Institute for Innovative Materials

2000

Analysis of linear and quadratic optical response of mixed LangmuirBlodgett films of stearic acid and 5-CT

Marc in het Panhuis

University of Wollongong, panhuis@uow.edu.au

R W. Munn

UMIST

Follow this and additional works at: https://ro.uow.edu.au/aiimpapers

Part of the Engineering Commons, and the Physical Sciences and Mathematics Commons

Research Online is the open access institutional repository for the University of Wollongong. For further information contact the UOW Library: research-pubs@uow.edu.au 


\title{
Analysis of linear and quadratic optical response of mixed Langmuir-Blodgett films of stearic acid and 5-CT
}

\author{
Abstract \\ Published experimental optical second-harmonic generation (SHG) results for mixed films formed \\ between stearic acid and the mesogen 5-CT (4"-n-pentyl-4-cyano- $p$-terphenyl) are reanalyzed. \\ Experimental refractive index results for the pure films are used to deduce molecular polarizabilities in \\ order to calculate local fields. At low and high fractions of 5-CT, a consistent interpretation of the SHG is \\ obtained using a mean molecular response based on a fixed axial hyperpolarizability $\beta$ LLL for the \\ mesogen, but at high mesogen fractions molecular tilt makes small off-diagonal \\ components $\beta_{\mathrm{LLM}} \approx-\beta_{\mathrm{LLL}} / 40$ essential. At intermediate fractions the treatment can interpret the SHG only \\ by requiring molecular tilt not found in the experiments, implying that a more detailed structural model is \\ required. \\ Keywords \\ optical, mixed, langmuir, films, stearic, acid, ct, linear, quadratic, response, blodgett, 5, analysis

\section{Disciplines} \\ Engineering | Physical Sciences and Mathematics \\ Publication Details \\ in het Panhuis, M. \& Munn, R. W. (2000). Analysis of linear and quadratic optical response of mixed \\ Langmuir-Blodgett films of stearic acid and 5-CT. Journal of Chemical Physics, 113 (23), 10691-10696.
}




\section{AD| $\begin{aligned} & \text { The Journal of } \\ & \text { Chemical Physics }\end{aligned}$}

\section{Analysis of linear and quadratic optical response of mixed Langmuir-Blodgett films of}

stearic acid and 5-CT

M. in het Panhuis and R. W. Munn

Citation: The Journal of Chemical Physics 113, 10691 (2000); doi: 10.1063/1.1323724

View online: http://dx.doi.org/10.1063/1.1323724

View Table of Contents: http://scitation.aip.org/content/aip/journal/jcp/113/23?ver=pdfcov

Published by the AIP Publishing

\section{Articles you may be interested in}

Second harmonic generation from copper-tetratert-butyl-phthalocyanine Langmuir-Blodgett film/metal interface: Electric quadrupole or electric field induced second harmonic generation effect?

J. Appl. Phys. 92, 6390 (2002); 10.1063/1.1518764

Phase-matched second harmonic generation and cascaded nonlinearity in a Langmuir-Blodgett inverted waveguide of 2-docosylamino-5-nitropyridine

J. Appl. Phys. 90, 5469 (2001); 10.1063/1.1412580

Microscopic treatment of substrate effects on linear and quadratic optical response of model Langmuir-Blodgett multilayers

J. Chem. Phys. 113, 10685 (2000); 10.1063/1.1323725

Optical anisotropy of Langmuir-Blodgett sapphyrin films

Appl. Phys. Lett. 77, 3164 (2000); 10.1063/1.1324983

Microscopic calculations of linear and quadratic optical response in model Langmuir-Blodgett multilayers J. Chem. Phys. 112, 6763 (2000); 10.1063/1.481238

\section{AlP hameded theses}

Journal of Applied Physics is pleased to announce André Anders as its new Editor-in-Chief 


\title{
Analysis of linear and quadratic optical response of mixed Langmuir-Blodgett films of stearic acid and 5-CT
}

\author{
M. in het Panhuis and R. W. Munn ${ }^{\text {a) }}$ \\ Department of Chemistry, UMIST, Manchester M60 1QD, United Kingdom
}

(Received 12 July 2000; accepted 19 September 2000)

\begin{abstract}
Published experimental optical second-harmonic generation (SHG) results for mixed films formed between stearic acid and the mesogen 5-CT (4"- $n$-pentyl-4-cyano- $p$-terphenyl) are reanalyzed. Experimental refractive index results for the pure films are used to deduce molecular polarizabilities in order to calculate local fields. At low and high fractions of 5-CT, a consistent interpretation of the SHG is obtained using a mean molecular response based on a fixed axial hyperpolarizability $\beta_{L L L}$ for the mesogen, but at high mesogen fractions molecular tilt makes small off-diagonal components $\beta_{L L M} \approx-\beta_{L L L} / 40$ essential. At intermediate fractions the treatment can interpret the SHG only by requiring molecular tilt not found in the experiments, implying that a more detailed structural model is required. (C) 2000 American Institute of Physics. [S0021-9606(00)70247-9]
\end{abstract}

\section{INTRODUCTION}

Langmuir-Blodgett films and other systems that comprise ordered molecular layers are of interest for developing materials with controllable structure and useful properties such as nonlinear optical response. Such materials are also often characterized optically, relying implicitly or explicitly on a suitable theory of the optical response. In previous publications, ${ }^{1,2}$ we presented a comprehensive microscopic treatment of linear and quadratic optical response in model Langmuir-Blodgett multilayers. This treatment took account of molecular tilt, of nonuniform distribution of polarizability and hyperpolarizability within the molecules, and of the influence of the substrate. In the present article, we illustrate the usefulness of the treatment by applying it to the specific example of optical second-harmonic generation (SHG) results reported ${ }^{3,4}$ for mixed monolayers formed between the classic Langmuir-Blodgett film forming species stearic acid and the molecule 5-CT, which exhibits a thermotropic liquid crystal phase (i.e., is a nematogen). The results were analyzed previously ${ }^{3}$ taking account of substrate effects by the method of images, but the molecules were treated as single points, whereas such elongated molecules imply the need for a submolecule treatment.

Measurements of linear and quadratic optical response were reported for mixed monolayers between stearic acid (SA: octadecanoic acid, structure 1) and each of three nematogens on a fused silica substrate. ${ }^{4}$ The most complete measurements involved the terphenyl 5-CT (4"-n-pentyl4-cyano- $p$-terphenyl, structure 2 , denoted in Ref. 4 by T15, but here denoted 5-CT following other work), ${ }^{5}$ on which we concentrate. The mixtures studied had molar compositions of $1 \%, 4 \%, 9 \%, 16 \%, 25 \%, 36 \%, 49 \%, 64 \%, 81 \%$ and $100 \%$ 5-CT. In the experiments involving SHG, the harmonic was close to a resonance frequency of 5-CT, so that results were reported in terms of the modulus of the quadratic susceptibility.

\footnotetext{
a) Author to whom correspondence should be addressed; electronic mail: R.W.Munn@umist.ac.uk
}

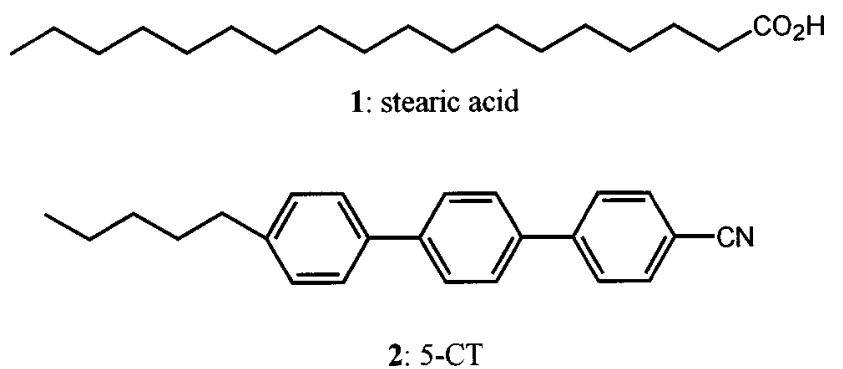

The results were analyzed previously, ${ }^{3}$ taking the substrate into account with the aid of a point-dipole model to deduce the local fields. Neither pure SA nor the substrate gave detectable SHG, leading to the conclusion that SHG from the mixtures originated exclusively from the hyperpolarizability of 5-CT. At the lower fractions of 5-CT, the only observable component of the surface susceptibility was $\left|\chi_{z z z}\right|$, where the $z$-axis is normal to the substrate. This indicates that 5-CT has a "one-dimensional" hyperpolarizability, in which the only numerically significant component is $\left|\beta_{L L L}\right|$, where $L$ is the long axis of the molecule (denoted the $\zeta$-axis previously). ${ }^{3}$ Such behavior is reinforced near a resonance, which again requires use of the modulus. The observation also shows that the molecules in these more dilute mixtures have their long axes normal to the substrate. At these concentrations the local-field effects were deduced to be negligible and an essentially constant value of $\left|\beta_{L L L}\right|$ was calculated from the observed values of $\left|\chi_{z z z}\right|$.

However, at the highest fractions of 5-CT a large component $\left|\chi_{z x x}\right|$ was observed, indicating that the molecules tilt markedly. If local-field effects are ignored, the calculated tilts are around $70^{\circ}$, but taking the local fields into account reduced the calculated tilts to about $50^{\circ}$. However, the apparent hyperpolarizability $\left|\beta_{L L L}\right|$ calculated from the observed values of $\left|\chi_{z z z}\right|$ fell markedly with increasing fractions of 5-CT, even when the tilt was apparently still zero, dropping by a factor of six for the pure 5-CT monolayer.

The marked variation in the calculated apparent hyper- 
polarizability clearly requires investigation, since one would expect the value to be at least roughly constant. As already noted, a point-dipole model was used for the local fields, which seems questionable for such elongated molecules, as the authors noted. ${ }^{3}$ Partly as a result, the treatment also failed to treat any effect of tilt on the local fields, so that the use of the local fields in deducing the tilt was not entirely selfconsistent. The treatment also used unmodified planewise dipole sums rather than the planewise Lorentz-factor tensors we use. ${ }^{1,2}$ This is equivalent to calculating the response to the applied optical electric field rather than the macroscopic field. Finally, the treatment neglected any local fields due to the SA molecules, on the grounds that they are much less polarizable than the 5-CT molecules. However, this effect is offset in the dilute mixtures by the much greater fraction of SA molecules. For example, in the $1 \%$ mixture, on average some ten SA molecules intervene between each pair of 5-CT molecules in any direction, so that the system is arguably better described as an isolated 5-CT molecule in pure SA.

Hence we have reanalyzed the results to explore the consequences that arise from using our more rigorous treatment. ${ }^{1,2}$ Preliminary calculations ${ }^{2}$ show that taking account of the fused silica substrates used for the experiments, with a relative permittivity $\epsilon_{s}=2.122,{ }^{3}$ produces changes of $3.5 \%$ in the perpendicular component of the local-field tensor $d_{z z}$ and $0.5 \%$ in the parallel component $d_{x x}$, even when a highly nonuniform distribution of response is assumed, and the changes reduce to $1 \%$ and $0.2 \%$, respectively for a uniform distribution. Substrate effects were reported to be very small in the previous work. ${ }^{3}$ We have no information on the actual distribution of response within the molecules, but since neither SA nor 5-CT possesses a very strong chromophore, while the conjugation in 5-CT extends over a sizeable fraction of the molecular length, we have for simplicity assumed that the distribution can adequately be approximated as uniform. Given this assumption, we have further assumed that substrate effects can be ignored in subsequent calculations. Also for simplicity we do not treat frequency dependence explicitly. Before treating the mixed films we need to treat the pure films, and we begin with pure stearic acid, which is simpler.

\section{ANALYSIS}

\section{A. Pure stearic acid}

Stearic acid gives no detectable SHG, but we argued above that it does contribute to the local field in mixed monolayers. Hence analysis is required to determine its polarizability. Since a mixed film that contains only $1 \%$ of 5-CT exhibits zero tilt, we assume that this holds also for pure SA. This assumption is supported by $\mathrm{x}$-ray measurements on metal stearate films, ${ }^{6}$ which also indicate a thickness of about $25 \AA$. Extrapolation of the reported surface number densities $N_{s}$ of the mixed films to zero 5-CT content gives an area per molecule of $19.8 \AA^{2}$, which yields a square lattice of side $4.44 \AA$. We treat the molecule as a set of ten submolecules equally spaced along its length, i.e., $2.5 \AA$ apart. The substrate is placed so that the real submolecule closest to it is also $2.5 \AA$ away from the image submolecule closest to it. For this case the planewise dipole sum (see Refs. 1 and 2) has nonzero components $T_{x x}=T_{y y}=0.4516$ and $T_{z z}=-0.9031$, so that the Lorentz-factor tensor (see Refs. 1 and 2) has $L_{x x}=L_{y y}=0.4516$ but $L_{z z}=0.0969$.

The relative permittivity $\epsilon_{r}$ of the pure SA film was reported $^{4}$ as 1.96. This value applies for propagation in the film, polarized normal to the substrate, and hence leads to the corresponding component of the polarizability of SA. This in turn can be identified as the axial $L L$-component, because the molecules are not tilted. From Eq. (15) in Ref. 2 we then obtain the polarizability volume $\alpha_{L L}$ as $34.5 \AA^{3}$. This value seems consistent with the value of about $19 \AA^{3}$ calculated for $n$-nonane, ${ }^{7}$ provided the carboxylic acid group does not produce any large enhancement. The value of $34.5 \AA^{3}$ relies on using the Lorentz-factor tensors; if the unmodified planewise dipole sums are incorrectly used instead, the unreasonably large value of $283 \AA^{3}$ results. Similarly, if the permittivity is assumed to be the value parallel to the substrate, the perpendicular component $\alpha_{M M}$ is obtained as $26.3 \AA^{3}$, which again appears too large compared with about $14 \AA^{3}$ calculated for $n$-nonane. ${ }^{7}$ Here $\alpha_{M M}$ is equal to $\alpha_{N N}$ under present approximations that imply axially symmetric molecules, where $M$ and $N$ are the two remaining molecular axes, denoted $\xi$ and $\eta$ previously. ${ }^{3}$

From the value of $34.5 \AA^{3}$ for $\alpha_{L L}$, we obtain the localfield tensor component $d_{z z}=1.09$. This modest value reflects the modest molecular polarizability in a large molecular volume, combined with the small value of $L_{z z}$. The anisotropic Lorentz local-field factor $\left(\epsilon_{r}+2\right) / 3$ yields $d_{z z}=1.32$, but is known to be unreliable for elongated molecules, ${ }^{8}$ for which the local-field factor approaches unity, the value appropriate to a needle-shaped Lorentz cavity. ${ }^{9}$ Taking $\alpha_{M M}$ as $14 \AA^{3}$ gives $d_{x x}=1.19$. Here the modest polarizability is reinforced by a larger Lorentz-factor tensor component to give a localfield component that is larger, though still not large in absolute terms.

\section{B. Pure 5-CT: Linear response}

For pure 5-CT the tilt indicated by the SHG measurements complicates the analysis. The tilt is required in order to define the structure and hence calculate the Lorentz-factor tensors. These are required in order to obtain the polarizability and hence the local fields, but the local fields are required to analyze the SHG measurements in order to deduce the tilt. In principle there is a self-consistent iterative solution, but we shall see that problems arise.

The first stage of the analysis is to deduce the polarizability and the local fields for a given tilt, which follows the procedure described for SA. The surface density of the 5-CT films gives an area per molecule of $28.8 \AA^{2}$, which yields a square lattice of side $5.37 \AA$, consistent with replacement of much of the alkyl chain in SA by the bulkier phenyl rings. Since the backbone of the molecule is 18 carbon atoms long (counting a phenyl ring as four carbons) like that in SA, we treat it as the same length as SA, $25 \AA$. We also treat it like $\mathrm{SA}$ as a set of ten submolecules equally spaced along its length, i.e., $2.5 \AA$ apart. The molecules are tilted by an angle $\theta$ away from the normal to the substrate in the $x z$-plane, so that the structure becomes monoclinic with monoclinic angle 
TABLE I. Axial polarizability components $\alpha_{L L} / \AA^{3}$ for 5-CT deduced for a range of tilt angles $\theta$ assuming $\alpha_{M M}=20 \AA^{3}$.

\begin{tabular}{lccccccccc}
\hline \hline Tilt, $\theta$ & $10^{\circ}$ & $20^{\circ}$ & $30^{\circ}$ & $40^{\circ}$ & $45^{\circ}$ & $50^{\circ}$ & $55^{\circ}$ & $60^{\circ}$ & $70^{\circ}$ \\
\hline$\alpha_{L L} / \AA^{3}$ & 63.2 & 59.5 & 55.3 & 51.9 & 50.8 & 50.0 & 49.5 & 48.6 & 36.7 \\
\hline \hline
\end{tabular}

$\beta=90^{\circ}+\theta$. The substrate is again placed so that the real submolecule closest to it is $2.5 \AA$ away from the image submolecule closest to it.

The relative permittivity $\epsilon_{r}$ of the pure 5-CT film was reported ${ }^{4}$ as 2.30, which as in pure SA applies to propagation in the film, polarized normal to the substrate. Because of the tilt, the polarizability normal to the substrate mixes the transverse polarizability $\alpha_{M M}$ in the plane of tilt with the axial polarizability $\alpha_{L L}$, which therefore cannot be deduced directly. To circumvent this problem, we fix a value for $\alpha_{M M}$ and obtain the value of $\alpha_{L L}$ then required to yield the observed $\epsilon_{r}$. In crystalline $p$-terphenyl, the mean polarizability perpendicular to the long axis was found ${ }^{10}$ to be $23 \AA^{3}$, which we round down to $20 \AA^{3}$ for 5-CT to take account of the smaller polarizability expected transverse to its alkyl chain.

We illustrate this procedure for a tilt of $45^{\circ}$. The Lorentz-factor tensor has independent nonzero components $L_{x x}=0.3508, L_{x z}=0.2922, L_{y y}=0.2355$, and $L_{z z}=0.4136$, which yield $\alpha_{L L}=50.8 \AA^{3}$. This value is some $50 \%$ larger than that deduced for SA, which appears plausible. It is also some $20 \%$ smaller than that deduced in crystalline $p$-terphenyl, ${ }^{10}$ which is less satisfactory, but there the molecule was represented as only three points, which would exaggerate the resulting polarizability. The local-field tensor corresponding to this polarizability is

$$
\mathbf{d}=\left(\begin{array}{ccc}
1.71 & 0 & 0.70 \\
0 & 1.09 & 0 \\
0.75 & 0 & 1.81
\end{array}\right)
$$

Compared with SA, this has two diagonal components much bigger than unity, consistent with the larger $\epsilon_{r}$ and larger polarizability. It also has sizeable off-diagonal components, which are allowed to be unequal.

The axial polarizability components deduced for a range of tilt angles are shown in Table I. The values decrease as the tilt increases, until for a tilt of $80^{\circ}$ no sensible value can be deduced with the chosen value of $\alpha_{M M}$. For $\alpha_{M M}=15$ or 10 $\AA^{3}$ we obtain $\alpha_{L L}=5.0$ or $16.4 \AA^{3}$, which are numerically sensible but physically much too small. This analysis leads to the conclusion that for reasonable polarizabilities to be deduced the tilt should be no greater than about $60^{\circ}$.

\section{Pure 5-CT: Nonlinear response}

The previous analysis of nonlinear response ${ }^{3}$ used the expression

$$
\tan ^{2} \theta=\left(\frac{d_{z z}}{d_{x x}}\right)^{2} \frac{2 \chi_{z x x}}{\chi_{z z z}}
$$

(where in the original work the mean tilt angle was called $\bar{\psi}$ and the local-field components were called $L_{z z}$ and $L_{x x}$ ). For convenience we define $R=2 \chi_{z x x} / \chi_{z z z}$. It can be seen that zero $\chi_{z x x}$ implies zero $R$ and zero tilt regardless of the local fields, but for nonzero $R$ deducing the tilt depends on the local fields. For pure 5-CT, ignoring the local-field correction led to $\theta=76^{\circ}$, but the calculated ${ }^{3}$ correction using $d_{z z} / d_{x x} \approx 0.3$ decreased $\theta$ significantly to $53^{\circ}$. However, the present calculations show that the ratio of these local fields in 5-CT is in fact close to unity for all tilts, so that in our treatment the tilt would not reduce much from $76^{\circ}$. Having $d_{z z} / d_{x x} \approx 1$ has the advantage that the observed value of $\chi_{z z z}$ no longer implies the drastic six-fold reduction in the apparent value of $\beta_{L L L}$ from that deduced for dilute mixtures where tilt is not an issue. (Henceforth for simplicity the modulus signs will not be written.) On the other hand, retaining a large value of $\theta$ gives rise to the problems of instability in deducing the polarizability already mentioned.

However, this difficulty need not be pursued, because the expression, Eq. (1), does not allow for the role of the off-diagonal components of the local-field tensor, which we have seen can be large. For example, $d_{x z}$ induces contributions to $\chi_{z z z}$ from the hyperpolarizability component $\beta_{x x x}$ parallel to the substrate. We have therefore calculated the ratio $R$ explicitly for a given tilt. Since the experiments show that the monolayers are isotropic in the $x y$-plane, ${ }^{4}$ we average $\chi_{z x x}$ and $\chi_{z y y}$ to obtain an effective value of $\chi_{z x x}$ for comparison with experiment. For the monoclinic structures we use, $\chi_{z y y}=0$ and so we calculate $R$ as just $\chi_{z x x} / \chi_{z z z}$. Unfortunately, this approach does not solve the difficulty over instability in deducing the polarizability, because the tilt required to reproduce the experimental value of $R$ (which we obtain as 19.6 from the quoted tilt and local-field factor) remains close to the starting value of $76^{\circ}$.

Such large tilts are required to reproduce the large observed value of $R$ because of the "one-dimensional" hyperpolarizability, which requires the long $L$-axis to lie much closer to the $x$-axis than to the $z$-axis. This one-dimensional hyperpolarizability implies that the dominant electronic transitions in 5-CT are polarized exclusively along the long axis. This in turn implies that the polarizability should also be one-dimensional, with no component perpendicular to the molecular axis, which conflicts with our assumption for the polarizability analysis. Presumably transitions polarized along the axis are dominant, while those polarized across the axis have much smaller but still nonzero transition dipoles. Since the hyperpolarizability depends on products of three transition dipoles while the polarizability depends on products of two, the hyperpolarizability can then be close to onedimensional while the polarizability is merely strongly anisotropic. However, this implies that the hyperpolarizability must have small but nonzero components that might nevertheless contribute significantly to the film response. In particular, given the dominance of transitions polarized along the $L$-axis, the next largest components after $\beta_{L L L}$ will be $\beta_{L L M}$ and its equivalents with one subscript different from $L$. Moreover, for zero tilt such components do not contribute to $\chi_{z x x}$, which can therefore remain zero as found experimentally.

We have therefore explored the effect of including these off-diagonal components of hyperpolarizability in our treat- 
TABLE II. Off-diagonal components $\left(\beta_{L L M} / 4 \pi \epsilon_{0}\right) / 10^{-40} \mathrm{~m}^{4} \mathrm{~V}^{-1}$ fitted to the observed susceptibility ratio $R=19.6$ for pure 5-CT for a range of tilt angles $\theta$, and resulting values of $\chi_{z z z} / 10^{-50} \mathrm{~m}^{2} \mathrm{~V}^{-1}$ (observed value 126).

\begin{tabular}{lrrrccccc}
\hline \hline Tilt, $\theta$ & $10^{\circ}$ & $20^{\circ}$ & $30^{\circ}$ & $40^{\circ}$ & $45^{\circ}$ & $50^{\circ}$ & $55^{\circ}$ & $60^{\circ}$ \\
\hline$\beta_{L L M}$ & -1035 & -379 & -177 & -86.6 & -59.6 & -40.3 & -26.6 & -17.4 \\
$\chi_{z z z}$ & 107 & 114 & 123 & 127 & 125 & 120 & 111 & 98 \\
\hline \hline
\end{tabular}

ment. For this purpose we adjust the ratio between the common value of these components and the value of $\beta_{L L L}$ so as to reproduce the observed value of $R$. We also take the opportunity to use absolute rather than relative values, taking the value $\beta_{L L L} / 4 \pi \epsilon_{0}=960 \times 10^{-40} \mathrm{~m}^{4} \mathrm{~V}^{-1}$ as deduced from the previous analysis ${ }^{3}$ for the most dilute mixed films, where there are no uncertainties caused by tilt and the local fields. This allows us to calculate the value of $\chi_{z z z}$ for comparison with the experimental value, which we estimate from the previous analysis ${ }^{3}$ to be $126 \times 10^{-50} \mathrm{~m}^{2} \mathrm{~V}^{-1}$. Note this is a surface susceptibility defined in terms of surface polarization expressed as dipole moment per area rather than volume. It differs from the volume susceptibility by a factor equal to the thickness of a monolayer. ${ }^{11}$

The results of this analysis are shown in Table II. For tilts of $40^{\circ}$ and above, the off-diagonal components are less than $10 \%$ of $\beta_{L L L}$, so that the response remains effectively one-dimensional. It is striking how small a value of $\beta_{L L M}$ suffices to fit $R$ at tilt angles where there is no problem with numerical instability in the polarizability. In principle comparison of the calculated values of $\chi_{z z z}$ with experiment could be used to determine the tilt, but the values vary surprisingly weakly with tilt. The only conclusion that may reasonably be drawn is that the tilt could lie rather lower than the range $50^{\circ}-75^{\circ}$ implied by the previous treatment. Perhaps more important is the fact that once the off-diagonal components are adjusted to fit the observed value of $R$, the observed value of $\chi_{z z z}$ for pure 5-CT is readily reproduced with the fixed value of $\beta_{L L L}$ derived from the dilute mixed films. There is no need to invoke a strongly varying apparent hyperpolarizability.

\section{Mixed monolayers}

We now test the integrity of the foregoing analysis by attempting to predict the properties of the mixed monolayers. For this purpose we introduce the variable $x$, the mole fraction of 5-CT in the mixture. We treat the monolayers as uniform arrays of composite molecules that consist of 5-CT and SA in suitable proportions. The molecules are already assumed to be the same length, and Lorentz-factor tensors are calculated using spacings between molecules obtained from the area per composite molecule calculated as $x / N_{s}$, where $N_{s}$ is the reported surface number density of 5-CT molecules in the monolayer of composition $x$. The composite molecules have properties obtained as weighted averages of those for the pure components. Hence the polarizability tensor is taken as

$$
\alpha(x)=x \alpha^{5-\mathrm{CT}}+(1-x) \alpha^{\mathrm{SA}},
$$

TABLE III. Off-diagonal components $\left(\beta_{L L M} / 4 \pi \epsilon_{0}\right) / 10^{-40} \mathrm{~m}^{40} \mathrm{~V}^{-1}$ for 5-CT fitted to the observed susceptibility ratio $R=8.0$ for a mixed film with $x=0.81$ for a range of tilt angles $\theta$, and resulting values of $\chi_{z z z} / 10^{-50} \mathrm{~m}^{2} \mathrm{~V}^{-1}$ (observed value 185).

\begin{tabular}{lcccccc}
\hline \hline Tilt, $\theta$ & $45^{\circ}$ & $50^{\circ}$ & $55^{\circ}$ & $57^{\circ}$ & $58^{\circ}$ & $60^{\circ}$ \\
\hline$\beta_{L L M}$ & -70.3 & -45.8 & -28.2 & -22.6 & -20.1 & -15.1 \\
$\chi_{z z z}$ & 380 & 218 & 199 & 189 & 182 & 171 \\
\hline \hline
\end{tabular}

where the superscripts denote the pure components. The hyperpolarizability tensor is taken as

$$
\beta(x)=x \beta^{5-\mathrm{CT}},
$$

since the hyperpolarizability of SA appears to be negligible, as shown by the linear dependence of $\chi_{z z z}$ on $x$ for small $x$ when SA molecules predominate. ${ }^{3}$ However, the value of $\beta_{L L M}$ was not precisely determined for pure 5-CT because fitting $R$ for different values of the tilt angle $\theta$ gave little variation in the other observed quantity $\chi_{z z z}$, as Table II shows. This does not matter for untilted films at small $x$, where this component does not affect $\chi_{z z z}$, but it does matter in the tilted films at large $x$. However, in these films it turns out that fitting $R$ for different values of tilt allows us to fix value of $\beta_{L L M}$ that fits both $R$ and $\chi_{z z z}$. Hence it is desirable to start the analysis on the films with large $x$.

For $x=0.81$, a procedure like that for pure 5-CT but with the molecular response given by Eqs. (2) and (3) yields the results shown in Table III. In this case $\chi_{z z z}$ varies significantly and allows estimates of the tilt angle $\theta$ and $\beta_{L L M} / 4 \pi \epsilon_{0}$ as about $57.5^{\circ}$ and $-21 \times 10^{-40} \mathrm{~m}^{4} \mathrm{~V}^{-1}$. For $x$ $=0.64$, the same procedure yields the results shown in Table $\mathrm{IV}$, with estimates of $\theta$ and $\beta_{L L M} / 4 \pi \epsilon_{0}$ as about $55^{\circ}$ and $-24 \times 10^{-40} \mathrm{~m}^{4} \mathrm{~V}^{-1}$. These results show that a consistent picture of these two mixtures and pure 5-CT can be obtained with tilts of $55^{\circ}-60^{\circ}$ and $\beta_{L L M} / 4 \pi \epsilon_{0}$ of about -24 $\times 10^{-40} \mathrm{~m}^{4} \mathrm{~V}^{-1}$. This is significant in showing that fixed values of $\beta_{L L L}$ and $\beta_{L L M}$ suffice to fit the observations. It also provides values that we can apply to the other mixtures.

For the remaining mixtures, the observations of $R$ clearly show zero tilt. The calculated values of $\chi_{z z z}$ are then given in Table $\mathrm{V}$ with the observed values. It can be seen that up to $x=0.09$ the calculated values are in excellent agreement, and for 0.16 the agreement may be considered acceptable. However, for higher values of $x$ the values do not agree at all. This disagreement reflects a conflict inherent in the observations. ${ }^{3,4}$ For these intermediate values of $x$ the tilt is deduced to be zero, which should make $\chi_{z z z}$ proportional to $x$, whereas $\chi_{z z z}$ is found not to be proportional to $x$, which for fixed hyperpolarizability should make the tilt nonzero.

TABLE IV. Off-diagonal components $\left(\beta_{L L M} / 4 \pi \epsilon_{0}\right) / 10^{-4} \mathrm{~m}^{4} \mathrm{~V}^{-1}$ for 5-CT fitted to the observed susceptibility ratio $R=4.9$ for a mixed film with $x$ $=0.64$ for a range of tilt angles $\theta$, and resulting values of $\chi_{z z z} / 10^{-50} \mathrm{~m}^{2} \mathrm{~V}^{-1}$ (observed value 232).

\begin{tabular}{lccc}
\hline \hline Tilt, $\theta$ & $50^{\circ}$ & $55^{\circ}$ & $60^{\circ}$ \\
\hline$\beta_{L L M}$ & -43.1 & -24.7 & -11.0 \\
$\chi_{z z z}$ & 260 & 233 & 200 \\
\hline \hline
\end{tabular}


TABLE V. Observed values of $\chi_{z z z} / 10^{-50} \mathrm{~m}^{2} \mathrm{~V}^{-1}$ for mixed films, values calculated for zero tilt, and tilt angles calculated to reproduce the observed values, with the resulting calculated values of $\chi_{z z z}$ and the susceptibility ratio $R$.

\begin{tabular}{lccccccc}
\hline \hline \multicolumn{1}{c}{$x$} & 0.01 & 0.04 & 0.09 & 0.16 & 0.25 & 0.36 & 0.49 \\
\hline$\chi_{z z z}$ (observed) & 44 & 173 & 386 & 653 & 697 & 759 & 759 \\
tilt & $0^{\circ}$ & $0^{\circ}$ & $0^{\circ}$ & $0^{\circ}$ & $0^{\circ}$ & $0^{\circ}$ & $0^{\circ}$ \\
$\chi_{z z z}$ (calculated) & 44 & 174 & 389 & 691 & 1078 & 1556 & 2132 \\
tilt & $0^{\circ}$ & $0^{\circ}$ & $0^{\circ}$ & $9^{\circ}$ & $27^{\circ}$ & $34^{\circ}$ & $40^{\circ}$ \\
$\chi_{z z z}$ (calculated) & 44 & 174 & 389 & 655 & 697 & 761 & 758 \\
$R$ (calculated) & 0 & 0 & 0 & 0.03 & 0.27 & 0.51 & 0.86 \\
\hline \hline
\end{tabular}

We have therefore explored the tilt angles required to reproduce the observed values of $\chi_{z z z}$ with $\beta_{L L M} / 4 \pi \epsilon_{0}$ set as $-24 \times 10^{-40} \mathrm{~m}^{4} \mathrm{~V}^{-1}$, with the results also shown in Table V. The observed values of $\chi_{z z z}$ are reproduced by steadily increasing values of tilt angle that join quite smoothly on to the tilt angles already deduced for the largest values of $x$. This agreement is secured at the cost of predicting values of the susceptibility ratio $R$ that approach unity but were in fact observed to be zero.

Finally, for completeness we record in Table VI the refractive indices calculated for all the films, including pure stearic acid and pure 5-CT. For tilted films, the refractive indices must reflect the observed axial symmetry. Hence a procedure has been adopted like that used for calculating the susceptibility ratio $R$. The linear susceptibility components $\chi_{x x}$ and $\chi_{y y}$ have been averaged to give a mean in-plane susceptibility $\chi_{x}$ from which the in-plane refractive index $n_{x}$ is obtained as $\triangleleft\left(1+\chi_{x}\right)$, while the out-of-plane refractive index $n_{z}$ is obtained as $\sqrt{ }\left(1+\chi_{z z}\right)$. Starting from pure SA, both refractive indices initially decrease with increasing 5-CT content, because the increasing area per molecule outweighs the effect of adding the more polarizable 5-CT molecules. This decrease continues for $n_{z}$ up to $50 \%$ 5-CT, but by $25 \% 5$-CT, $n_{x}$ has started increasing as the effects of tilt and increasing average polarizability reinforce one another.

\section{DISCUSSION AND CONCLUSIONS}

We have applied the methods of our two previous articles $^{1,2}$ to analyze results reported in the literature ${ }^{3,4}$ for mixed monolayers formed between stearic acid and the terphenyl 5-CT. Aspects of the previous treatment have been corrected by using planewise Lorentz-factor tensors instead of unmodified dipole sums and by allowing stearic acid to contribute to the local fields. The experimental results cannot be reproduced if the hyperpolarizability of 5-CT is fixed in value and strictly one-dimensional, except for small fractions of 5-CT. Introducing very small fixed off-diagonal components (fixed at only $1 / 40$ of the magnitude of the single nonzero diagonal component, and with opposite sign) allows us to reproduce the results at large fractions of 5-CT as well, and yields plausible values for the molecular tilt away from vertical. The experimental results for intermediate fractions of 5-CT are still not reproduced by our approach using composite molecules unless the molecules are allowed to tilt, whereas the experiments would certainly have detected tilts as large as those calculated here. However, the information deduced from the experimental results ${ }^{3}$ and the conclusions from our approach would be affected if other susceptibility components induced by modest tilt offset the observable effect of $\chi_{z x x}$.

The discrepancy could perhaps arise from some structural feature of these films, such as domain formation in this region where the proportions of stearic acid and 5-CT are comparable. Another possibility is formation of a different phase at intermediate fractions: in neat 5-CT there is spectroscopic evidence ${ }^{12}$ for different crystalline phases at different temperatures. In pure 5-CT films vacuum-deposited on solid substrates, the molecules appeared to show nearly zero tilt, with probable formation of $\mathrm{H}$ aggregates, as opposed to the significant tilt in Langmuir-Blodgett deposited films of pure 5-CT. ${ }^{3}$ However, atomic force microscope studies showed changes in the crystalline morphology of the vacuum-deposited films from small plates to needles on standing, with evidence of increase in the tilt. ${ }^{12}$ Modeling of the structures of the mixed films would be helpful to clarify this behavior.

A recent article $^{13}$ analyzed the variation of the SHG intensity from a monolayer of the biphenyl analogue of 5-CT as it is compressed, using a rigid-rod model to relate the average molecular tilt to the area per molecule. Local field effects were not treated and a fixed one-dimensional molecular hyperpolarizability was assumed. For the single polarization chosen, the model reproduced qualitatively the marked increase in SHG intensity observed on compression, but no detailed quantitative comparison was presented. This work supports the idea that a fixed molecular hyperpolarizability should be adequate to model SHG, but naturally cannot provide insight into the structure of mixed films.

Our treatment takes no explicit account of the frequency dependence and in particular of the resonant absorption at the harmonic frequency. Thus we have deduced molecular polarizabilities from film permittivities around the nonresonant fundamental frequency $\omega$ and used these to calculate one set of local-field components rather than sets of components at both $\omega$ and $2 \omega$. This distinction could be particularly important when tilt is significant. ${ }^{3}$

The methods presented here permit rigorous and detailed

TABLE VI. Calculated refractive indices for pure and mixed films. The values in parentheses were used as input data.

\begin{tabular}{cccccccccccc}
\hline \hline$x$ & 0 & 0.01 & 0.04 & 0.09 & 0.16 & 0.25 & 0.36 & 0.49 & 0.64 & 0.81 & 1 \\
\hline Tilt & $0^{\circ}$ & $0^{\circ}$ & $0^{\circ}$ & $0^{\circ}$ & $9^{\circ}$ & $27^{\circ}$ & $34^{\circ}$ & $40^{\circ}$ & $55^{\circ}$ & $57^{\circ}$ & $58^{\circ}$ \\
$n_{x}$ & 1.195 & 1.191 & 1.182 & 1.171 & 1.164 & 1.185 & 1.197 & 1.215 & 1.275 & 1.320 & 1.401 \\
$n_{z}$ & $(1.400)$ & 1.395 & 1.382 & 1.363 & 1.347 & 1.343 & 1.334 & 1.332 & 1.339 & 1.393 & $(1.517)$ \\
\hline \hline
\end{tabular}


analysis of experimental results for linear and quadratic optical properties of Langmuir-Blodgett films. Applying such an analysis to films formed between stearic acid and the terphenyl 5-CT reveals effects that go beyond those expected from the simple one-dimensional model of molecular response. In particular, quite tiny deviations from strictly onedimensional response can have apparently disproportionate effects on the film response for tilted molecules. These effects are mediated by the sizeable off-diagonal components of the local-field tensor in such structures. Such components are often neglected for simplicity or through oversight, whereas a more reliable simple assumption in tilted films would be that the local-field tensor remains diagonal in the molecular axes rather than the film axes.

Since the rigorous analysis requires additional calculations but derives much the same molecular tilts as the previous analysis, ${ }^{3}$ one might ask what benefit is derived from the extra effort. The main benefit is that the molecular hyperpolarizability can remain fixed in value for the mixtures most concentrated in 5-CT, whereas previously it had to vary by a factor of six. Fixed molecular response accords better with physical intuition, and leads to experimentally verifiable predictions that could probe the discrepancies that remain for intermediate compositions. For this purpose it would be especially helpful if films could be prepared with large domains within which response could be studied without any averaging over orientations.

In conclusion, we believe that the analysis in this article shows that our approach ${ }^{1,2}$ provides a credible detailed qualitative and quantitative account of linear and nonlinear optical response in Langmuir-Blodgett films, and by implication in other molecular thin films. The treatment does require more input than most previous analyses, but it yields correspond- ingly more output. In particular it shows the limitations of the convenient one-dimensional approximation for molecular response once molecular tilt becomes significant. For mixed films at intermediate compositions the treatment can interpret the SHG only by requiring molecular tilt not found in the experiments. However, this is a limitation not of our general approach but rather of our simple model for the film as a uniform array of composite molecules, which therefore lacks the detailed structural information apparently required when neither molecule predominates.

\section{ACKNOWLEDGMENTS}

We are grateful to J. F. McGilp for encouraging discussions. This work was supported under the EU TMR Network Program, contract FMRX-CT96-0047.

\footnotetext{
${ }^{1}$ M. in het Panhuis and R. W. Munn, J. Chem. Phys. 112, 6763 (2000).

${ }^{2}$ M. in het Panhuis and R. W. Munn, J. Chem. Phys. 113, 10685 (2000), preceding paper.

${ }^{3}$ Z.-R. Tang, M. Cavanagh, and J. F. McGilp, J. Phys.: Condens. Matter 5, 3791 (1993); 5, 7903 (1993).

${ }^{4}$ Z.-R. Tang and J. F. McGilp, J. Phys.: Condens. Matter 4, 7965 (1992).

${ }^{5}$ G. Berkovic, T. Rasing, and Y. R. Shen, J. Opt. Soc. Am. B 4, 945 (1987).

${ }^{6}$ M. C. Petty, in Langmuir-Blodgett Films, edited by G. G. Roberts (Plenum, New York, 1990), p. 189.

${ }^{7}$ A. J. Stone, C. Hättig, G. Jansen, and J. G. Ángyán, Mol. Phys. 89, 595 (1996).

${ }^{8}$ J. H. Meyling, P. J. Bounds, and R. W. Munn, Chem. Phys. Lett. 51, 234 (1977).

${ }^{9}$ R. W. Munn, Synth. Met. 64, 117 (1994).

${ }^{10}$ P. J. Bounds and R. W. Munn, Chem. Phys. 24, 343 (1977).

${ }^{11}$ R. W. Munn, J. Chem. Phys. 103, 850 (1995).

${ }^{12}$ T. Sumiyoshi, I. Takahashi, Y. Tsuboi, H. Miyasaka, A. Itaya, T. Asahi, and H. Masuhara, Thin Solid Films 370, 285 (2000).

${ }^{13}$ M. Iwamoto, C.-X. Wu, and O.-Y. Zhong-can, Chem. Phys. Lett. 325, 545 (2000).
} 